\title{
Nonlinear resonant tunnelling through double-barrier structures
}

\author{
Enrique Diez ${ }^{a}$, Francisco Domínguez-Adame ${ }^{b}$, Angel Sánchez ${ }^{a}$ \\ ${ }^{a}$ Escuela Politécnica Superior, Universidad Carlos III de Madrid, c./ Butarque 15, E-28911 Leganés, Madrid, Spain \\ b Departamento de Física de Materiales, Facultad de Físicas, Universidad Complutense, E-28040 Madrid, Spain
}

Received 29 November 1994; revised manuscript received 17 January 1995; accepted for publication 18 January 1995

Communicated by A.R. Bishop

\begin{abstract}
We study resonant tunnelling through double-barrier structures under an applied bias voltage, in which nonlinearities due to self-interaction of electrons in the barrier regions are included. As an approximation, we concern ourselves with thin barriers simulated by $\delta$-function potentials. This approximation allows for an analytical expression of the transmission probability through the structure. We show that the typical peaks due to resonant tunneling decrease and broaden as nonlinearity increases. The main conclusion is that nonlinear effects degrade the peak-to-valley ratio but improve the maximum operation frequency of the resonant tunnelling devices.
\end{abstract}

Resonant tunnelling (RT) through a heterostructure quantum well has recently attracted considerable attention because of its applications in ultra-highspeed electronic devices. As an example, RT in GaAs/GaAlAs double-barriers at $\mathrm{THz}$ frequencies has been reported in the literature [1]. RT is a basic phenomenon which can be explained with elementary quantum mechanics [2]: there exists a dramatic increase of the transmission probability whenever the energy of an incident electron matches one of the unoccupied quasi-bound states inside the well. The energy of the incident electron is close to the Fermi energy, which is constant in the device. Therefore, to obtain a RT current in practice, one must modify the energy of the discrete level in the well applying a bias voltage. If initially there are no electrons in the well and the electrons are forced to tunnel by the applied voltage, after a time of a few $\sim \hbar / \Gamma, \Gamma$ being the width of the discrete level, high transmission is achieved by multiple reflection. This mechanism is physically analogous to what occurs in a Fabry-
Perot resonator. Since multiple scattering of waves is required for RT, it can be realized that such a phenomenon depends crucially on the linear superposition principle. Then a natural question arises in this context, namely how spatial nonlinearities affect RT. Nonlinearity may arise in semiconductors when electrons polarize the surrounding medium, which reacts changing the electron state by a feedback process, or when electron-electron interaction is taken into account. There are two important magnitudes which, in principle, could be largely modified by nonlinearity: the peak-to-valley ratio and the level width $\Gamma$, which is related to the reliability of the device at high frequencies. For instance, several satellite peaks in addition to the main peak have recently been found via coupled electron-phonon states in RT double-barrier heterostructures [3]. Moreover, the occurrence of nonlinearities opens the possibility of studying new and interesting phenomena in semiconductor devices, like bistability or chaotic wavefunctions [4]

In this Letter we focus our attention on a simple 
model to study nonlinear effects in double-barrier heterostructures. We consider very narrow barriers of a nonlinear material embedded in a linear material. We simulate the two barriers by means of $\delta$-function potentials, which allow for an analytical treatment even in the presence of nonlinearities. Similar models have been proposed to study wave propagation in nonlinear periodic media [5] and nonlinear Stark-Wannier resonances [6]. Thus, the propagation of a stationary wave in the nonlinear double-barrier heterostructure is described by means of the following Schrödinger equation (we use units such that $\hbar=m=1$ ),

$$
\begin{aligned}
& -\Psi^{\prime \prime}(x)+V(x) \Psi(x) \\
& \quad+[\delta(x)+\delta(x-a)]\left[\beta+\tilde{\alpha} \Psi(x) \Psi^{*}(x)\right] \Psi(x) \\
& \quad=E \Psi(x) .
\end{aligned}
$$

The parameter $\beta$ is the strength of the $\delta$-potential in the linear case (i.e. the area of the semiconductor barrier) and $\tilde{\alpha}$ measures the strength of the nonlinear coupling. The width of the quantum well is denoted by $a$. The potential due to the applied bias is $V(x)$. This potential may be simulated by step functions, which have been demonstrated to be a good approach to linearly rising potentials, reproducing accurately the properties of the electronic states, even the subtle ones [7]. Therefore we take

$$
\begin{aligned}
V(x) & =0, & & x<0, \\
& =-\frac{1}{2} V, & & 0<x<a, \\
& =-V, & & x>a,
\end{aligned}
$$

where $V$ is the applied voltage.

The solution of Eq. (1) in the linear semiconductor is a combination of travelling waves. As usual in scattering problems, we assume an electron incident from the left and evaluate the transmission and reflection probabilities when passing through the structure. To this end we take

$$
\begin{aligned}
\Psi(x) & =A\left(\mathrm{e}^{\mathrm{i} k_{0} x}+r \mathrm{e}^{-\mathrm{i} k_{0} x}\right), & & x<0, \\
& =A\left(C \mathrm{e}^{\mathrm{i} k_{1} x}+D \mathrm{e}^{-\mathrm{i} k_{1} x}\right), & & 0<x<a, \\
& =A t \mathrm{e}^{\mathrm{i} k_{2} x}, & & x>a .
\end{aligned}
$$

$t$ and $r$ denote the transmission and reflection amplitudes, and we have defined for simplicity $k_{0}^{2}=E, k_{1}^{2}=$

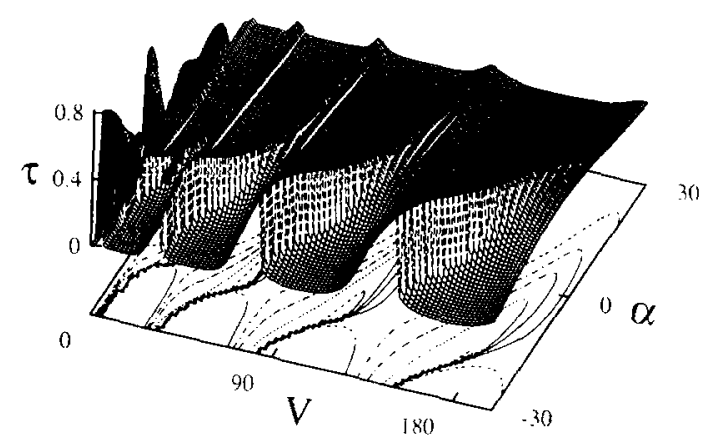

Fig. 1. Transmission probability $T$ as a function of the applied voltage $V$ and the nonlinear coupling $\alpha$ for $E=2, \beta=10$, and $a=1.5$.

$E+\frac{1}{2} V$, and $k_{2}^{2}=E+V$. The transmission probability is then found to be $T=\left(k_{0} / k_{2}\right)|t|^{2}$. To compute it we must set appropriate boundary conditions at the barriers, in order to eliminate the two unknowns $C$ and $D$. These can be obtained by integrating Eq. (1) around $x=0$ and $x=a$. In so doing, we find that the wave function itself is continuous whereas its derivative satisfies

$$
\begin{aligned}
& \Psi^{\prime}\left(0^{+}\right)-\Psi^{\prime}\left(0^{-}\right)=\left[\beta+\tilde{\alpha}|\Psi(0)|^{2}\right] \Psi(0), \\
& \Psi^{\prime}\left(a^{+}\right)-\Psi^{\prime}\left(a^{-}\right)=\left[\beta+\tilde{\alpha}|\Psi(a)|^{2}\right] \Psi(a)
\end{aligned}
$$

Using these boundary conditions and solution (3) we have after a little algebra

$$
\begin{aligned}
r= & t\left(\cos \left(k_{1} a\right)+\frac{k_{1}}{\sin \left(k_{1} a\right)}\left[\left(\beta+\alpha|t|^{2}\right)-\mathrm{i} k_{2}\right]\right) \\
& -1, \\
t= & (1+r) \frac{\sin \left(k_{1} a\right)}{k_{1}} \\
& \times\left\{\left[k_{1} \cot \left(k_{1} a\right)+\mathrm{i} k_{0}+\beta+\alpha|1+r|^{2}\right]-2 \mathrm{i} k_{0} r\right\},
\end{aligned}
$$

where $\alpha \equiv \tilde{\alpha}|A|^{2}$. Finally, inserting (5a) in (5b) we obtain an expresion for the transmission amplitude, and thus the transmission probability can be found for a given set of parameters $(E, \alpha, \beta, a$ and $V)$.

Fig. 1 shows the transmission probability as a function of the applied voltage $V$ and the nonlinear coupling $\alpha$. The energy of the particle is taken to be $E=$ $2, \beta=10$, and $a=1.5$. It is important to mention that the qualitative aspects of the results remain almost unchanged when varying the parameters $E, \beta$, 

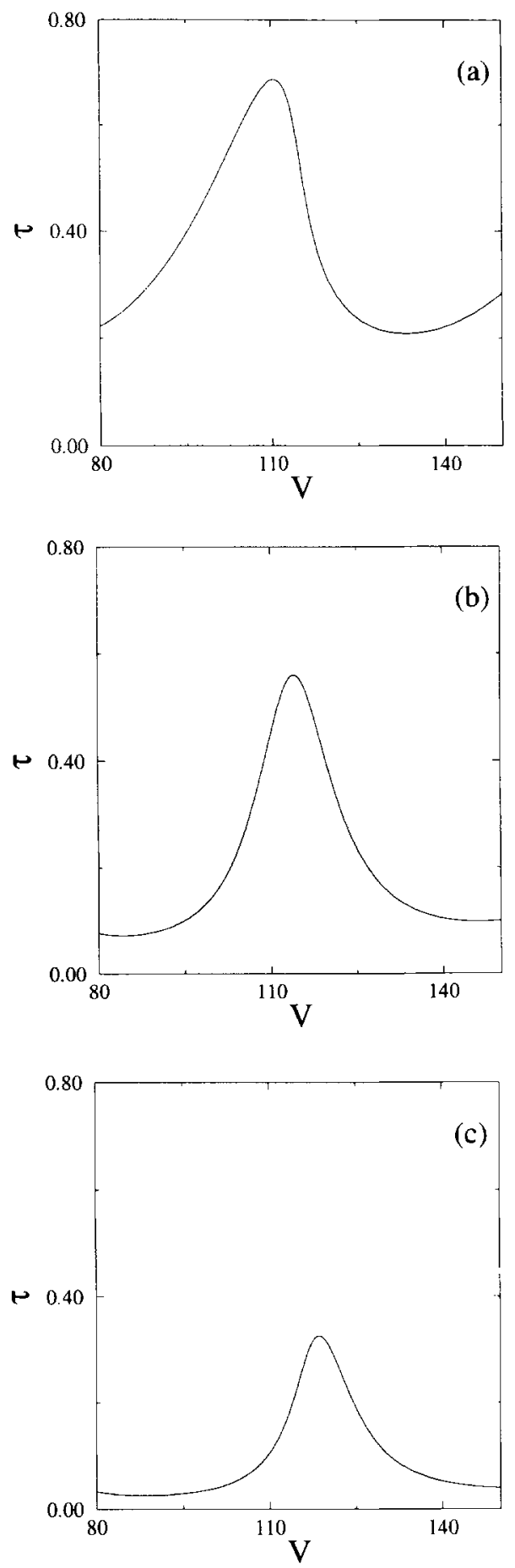

Fig. 2. Transmission probability $T$ as a function of the applied voltage $V$ for $E=2, \beta=10, a=1.5$, and (a) $\alpha=-10$, (b) 0 , and $(c)+10$. and $a$. From this plot it is clear that several peaks appear on increasing the applied voltage, independent of the value of the nonlinear coupling $\alpha$. Each peak corresponds to a quasi-bound state level in the well. It is also apparent that the width of these peaks is relatively large, specially for higher order ones. At this point we should remark that the width of the peaks appearing in Fig. 1 (transmission probability versus applied voltage) is not exactly equal to $\Gamma$, i. e., the level width. In addition, we note that a rigorous definition of $\Gamma$ requires the complicated expression ( $5 b$ ) to be a Lorentzian when approximated around each resonance. This is a cumbersome calculation which we have not attempted, but rather, we have numerically fitted Lorentzian shapes to our transmission coefficient curves with good agreement. Therefore, we feel confident that the following procedure gives a close approximation to $\Gamma$ : Assuming that the lifetime is not strongly dependent on the position of the level (so that it remains almost unchanged under minor variations of the applied voltage), it is clear that the width of the resonance peak in the transmission coefficient is very close to $\Gamma$. Hence we can accurately determine $\Gamma$ from Fig. 1 . The fact that $\Gamma$ is relatively large is easily understood by considering that the barriers are, in fact, very narrow, so that the coupling of the quantum well states with the continuum is very strong. Thus the lifetime is very short and, accordingly, $\Gamma$ is large. Also $\Gamma$ increases upon increasing the order of the quasi-bound state in the well, as expected. Now let us discuss the main effects of nonlinearity. In Fig. 1 one can see that the peak-to-valley ratio decreases dramatically on increasing $\alpha$ from -30 up to 30 . Moreover, it is also clear that the resonances shift to higher voltages when increasing $\alpha>0$ but, on the contrary, resonances shift to lower voltages for $\alpha<0$. This means that the quasi-bound states within the well region are raised on increasing nonlinearity. With more elaborate models, a similar shift to higher voltages whenever Coulomb repulsion between electrons increases in quasi-one-dimensional systems has been recently found [8]. Hence we are led to the conclusion that our model retains most of the physics of resonant tunneling, since Coulomb repulsion can be regarded as a possible origin of nonlinearities within the one-particle approach.

Since the width of the peaks is a very important parameter for the design of high-speed devices, we have 
also studied $\Gamma$ as a function of the nonlinear parameter. Results are shown in Fig. 2 for three different values of the nonlinear parameter $\alpha$. Besides the relative shift of the peaks mentioned above, it is seen that $\Gamma$ decreases with $\alpha$ for peaks of the same order. Hence we are led to the conclusion that, in order to improve the quality of the RT device, one should look for a compromise between the peak-to-valley ratio which decreases with $\alpha$, and the width of the levels which also decreases. Hence, on increasing nonlinearity, one obtains a lower RT current but, in principle, a higher operation frequency.

A possible extension of this work concerns narrowgap semiconductors for RT $[9,10]$. In this case RT takes place by an interband tunnelling mechanism, and a simple one-band description is no longer valid. Instead, one must use a more elaborate band structure including the effects of several bands of the host semiconductors. One of the simplest models is the two-band model [11], in which envelope functions of the conduction- and valence-bands are included. The equation of motion is then analogous to the Dirac equation for relativistic electrons. Hence, Eq. (1) should be replaced by a Dirac-like equation with nonlinear terms. Fortunately, the nonlinear Dirac equation with $\delta$-function potentials is also exactly solvable [12] and, therefore, the generalization of the present work to narrow-gap heterostructures seems to be achievable. In addition, more realistic and elaborate models are indeed required to describe nonlinear electron dynamics through thick barriers even if only oneenvelope function is used, for which the $\delta$-function approach is no longer valid. Work in this direction is in progress.

F.D.-A. acknowledges support from UCM through project PR161/93-4811. A.S. acknowledges partial support from C.I.C. y T. (Spain) through project PB92-0248 and from the European Union Human Capital and Mobility Programme through contract ERBCHRXCT930413.

\section{References}

[1] T.C.L.G. Sollner, W.D. Goodhue, P.E. Tannenwald, C.D. Parker and D.D. Peck, Appl. Phys. Lett. 43 (1984) 588.

[2] B. Méndez and F. Domínguez-Adame, Am. J. Phys. 62 (1994) 143

[3] T, Figielski, A. Mąkosa, T. Wosiński, P.C. Hamess and K.E. Singer, Solid State Commun. 91 (1994) 913

[4] P. Hawrylak, M. Grabowski and P. Wilson, Phys. Rev. B 40 (1989) 6398

[5] M. Grabowski and P. Hawrylak, Phys. Rev. B 41 (1990) 5783.

[6] E. Cota, J.V. José and G. Monsivais, J. Phys. 25 (1992) L57.

17] F. Bentosela, V. Grecchi and F. Zironi, J. Phys. C 15 (1982) 719.

[8] S. Nonoyama, A. Oguri, Y. Asano and S. Makeawa, Phys. Rev. B 50 (1994) 2667.

19] T.C. Gill and D.A. Collins, Semicond. Sci. Technol. 5 (1990) S1.

[10] R. Beresford, L.F. Luo and W.I. Wang, Semicond. Sci. Technol. 5 (1990) S195.

[11] J. Callaway, Quantum theory of the solid state (Academic Press, New York, 1991).

[12] F. Domínguez-Adame, J. Phys. A 26 (1993) 3863. 